\title{
METAPARENTAGEM: UMA NOVA POSSIBILIDADE DE AVALIAR A PARENTAGEM ${ }^{12}$
}

\author{
Sabrina Mazo D’Affonseca* \\ Lúcia Cavalcanti de Albuquerque Williams
}

\begin{abstract}
RESUMO. No presente trabalho objetiva-se apresentar um novo constructo cognitivo para analisar o relacionamento entre pais e filhos: a metaparentagem, definida como um conjunto de processos encobertos que resultam em uma compreensão mais abrangente da parentalidade, de modo que a metaparentagem consiste em pensar ou refletir a respeito das próprias práticas parentais. Em uma revisão da literatura realizada com as palavras-chaves metaparenting e metaparentagem em bases de dados nacionais e internacionais (PsycINFO, Lilacs, Sage, Medline e Google acadêmico), foram encontrados dois artigos, duas dissertações de mestrado e uma tese de doutorado, todos em língua inglesa. Por ser uma área de pesquisa recente, poucos estudos foram desenvolvidos na literatura internacional e nacional; contudo os dados obtidos demonstram que a metaparentagem foi correlacionada a parentagem democrática, competência parental e menor potencial de abuso, e parece ser um recurso útil para a área de intervenção em famílias e prevenção de problemas de comportamento infantil. Estudos com uma população maior, de diferentes culturas e níveis socioeconômicos e educacionais diversos contribuirão para a compreensão do fenômeno.
\end{abstract}

Palavras-chave: Relações pais-filhos; cognições; prevenção.

\section{METAPARENTING: A NEW POSSIBILITY TO EVALUATE PARENTING}

\begin{abstract}
This study presents a new cognitive construct to analyze parent-child relationship: metaparenting. This construct is defined as a set of processes that results in a more comprehensive understanding of parenting; metaparenting consists in thinking about or reflecting by parents on their own parenting practices. In a literature review conducted with the following keywords metaparenting and metaparentagem in national and international databases (PsycINFO, Lilacs, Sage, Medline and Google scholar), two studies were found, two master thesis and a doctoral dissertation, all of them in English. As an area of recent research, few studies have been developed in Brazilian and international literature, nevertheless the data obtained illustrates that metaparenting was correlated with authoritative parenting, parental competence and less potential for child abuse. Therefore it seems to be a useful resource for the area of intervention with families and preventing child behavior problems. Further studies with a larger population, from different cultures, different economics and educational levels should contribute for further understanding of the phenomenon.
\end{abstract}

Key words: Parent-child relationship; cognition; prevention.

\section{METAPARENTAGEM: UNA NUEVA POSIBILIDAD DE EVALUACIÓN DE COMO LOS PADRES EDUCAN A SUS NIÑOS}

RESUMEN. Este estudio objetiva presentar un nuevo construct cognitivo para examinar la relación entre padres e hijos la metaparentagem - que se define com un conjunto de procesos internos que llevan a una compreensión más integral de la parentalidad, por lo que la metaparentagem envuelve lo pensar, lo reflexionar sobre las propias prácticas parentales. En una revisión de literatura llevada a cabo con las siguientes palabras clave metaparentagem y metaparenting en bases de datos nacionales e internacionales (PsycINFO, LILACS, Sage, Medline y Google Scholar), se encontraron dos artículos, dos disertaciones de mestrado y una tesis doctoral, todos en Inglés. Siendo un área de

\section{Apoio Financeiro: Capes/CNPq}

O trabalho é parte da tese de doutorado da primeira autora.

Doutora em Psicologia pela Universidade Federal de São Carlos.

\# Doutora em Psicologia (Psicologia Experimental) pela Universidade de São Paulo. Professora titular da Universidade Federal de São Carlos, Brasil e coordenadora do Laboratório de Análise e Prevenção da Violência Laprev. 
investigacion reciente, pocos estudios se han desarrollado en la literatura brasilena o internacional. Sin embargo, los datos obtenidos demostram que la metaparentagem se correlaciono con la paternagen autoritativa, la competencia parental y un menor potencial de abuso. El constructo parece ser un recurso útil para el área de intervención con las familias y la prevención de problemas de comportamiento infantil. Otros estudios con una población, más grande, de diferentes culturas y niveles económicos y educativos distintos contribuirao para comprender el fenomeno en más profundidad.

Palabras-clave: Relación padres- hijos; cognición; prevención.

A família é o primeiro grupo social e a primeira forma de relação social da criança. Assim, delega-se a ela um papel central no desenvolvimento das pessoas, uma vez que, durante muitos anos, é o principal ambiente no qual a criança está inserida e, além disso, age como chave ou filtro que determina a abertura da criança a outros contextos (Papalia, Olds \& Feldman, 2009).

No contexto familiar o indivíduo tem sua sobrevivência física garantida e realiza as aprendizagens básicas necessárias a seu desenvolvimento dentro da sociedade (aprendizagem do sistema de valores, da linguagem, do controle da impulsividade, etc.). Por meio de diferentes mecanismos (por exemplo, recompensas e castigos recebidos pela criança; observação e imitação de comportamentos de outros familiares; identificação com outros membros da família), a criança desenvolve determinadas características psicológicas. Além disso, o relacionamento familiar tem 0 papel central de construir 0 autoconceito e a autoestima da criança, além de ter um impacto muito significativo nos seus comportamentos sociais, éticos, morais e cívicos. Em uma organização familiar saudável os pais apresentam estilos parentais baseados no uso de contingências positivas, o que favorecerá a aquisição de comportamentos prósociais e a formação de um autoconceito adequado dos filhos (Padilha \& Williams, 2004).

Guralnick (1998) aponta algumas características das relações familiares que promovem 0 desenvolvimento saudável das crianças: (a) responder contingentemente; (b) estabelecer reciprocidade; (c) promover afetividade e interações não instrutivas; (d) estruturar adequadamente o ambiente; (e) usar explicações verbais para corrigir "falhas"; (f) criar exigências e expectativas adequadas para a fase do desenvolvimento da criança nas interações cuidador-criança.

Devido à complexidade dos relacionamentos familiares e à importância de compreender as variáveis responsáveis pelo desenvolvimento saudável das crianças, as pesquisas na área do desenvolvimento infantil e família apresentadas por Papalia, Olds e Feldman (2009) selecionaram alguns aspectos que podem influenciar essa relação, entre os quais se destacam: (1) o ambiente, ou seja, o contexto em que ocorre a interação e a fase do desenvolvimento da criança; (2) 0 comportamento, as ações dos pais em relação aos cuidados da criança; e (3) as cognições, pensamentos e crenças dos pais e sua influência no comportamento das crianças.

Inicialmente as pesquisas (por exemplo, Baumrind, 1966, citada em Weber, Prado, Viezzer \& Brandenburg, 2004) se direcionaram aos comportamentos dos pais em relação aos filhos e a influência dos pais sobre o desenvolvimento saudável das crianças. Essa área de pesquisa utiliza o conceito de práticas educativas parentais, as quais são definidas por Gomide (2003) como as diversas estratégias e técnicas das quais os pais se utilizam para orientar os comportamentos dos filhos e cumprir seu papel como agentes de socialização, as quais são o conjunto de práticas educativas denominado estilos parentais. Para Gomide (2003), algumas práticas parentais utilizadas na educação dos filhos podem inibir a formação de comportamentos inadequados ou antissociais (monitoria positiva e comportamento moral) ou aumentar a ocorrência de problemas de comportamento em crianças (negligência, abuso físico e psicológico, disciplina relaxada, punição inconsistente e monitoria negativa).

Em uma revisão de literatura realizada por Tucunduva e Weber (2008) a respeito da interação e fatores familiares que representam proteção e risco para o desenvolvimento de crianças e adolescentes, notou-se que os conflitos familiares, a punição física, o afeto e o envolvimento parental são, respectivamente, os fatores de risco e proteção mais citados na literatura. Neste mesmo sentido, Bolsoni-Silva e Marturano (2002) demonstraram que o ambiente 
familiar e as práticas educativas parentais são fatores que podem tanto promover 0 desenvolvimento de comportamentos socialmente adequados como favorecer o surgimento de comportamentos problemáticos. Em uma pesquisa que comparou práticas educativas parentais e comportamentos de crianças de um grupo de crianças considerado clínico/com problema de comportamento $(n=27)$ com um grupo de crianças não clínico/sem problema de comportamento $(n=26)$, observouse que uma das variáveis que diferenciavam os grupos era as práticas educativas positivas utilizadas pelos pais (Bolsoni-Silva \& Loureiro, 2011).

Salvador e Weber (2008) observaram que a promoção de comportamentos pró-sociais e saudáveis (competência social, estratégias adequadas de enfrentamento, autoestima, autoconceito e autoeficácia elevados) era decorrente de interações familiares positivas, as quais incluíam, especialmente, afetividade, responsividade, envolvimento e uso contingente de reforçadores positivos. Em contraste, os prejuízos ao desenvolvimento de crianças eram decorrentes da ausência dessas práticas ou de seu uso inadequado. As autoras destacaram haver associação entre falta de disciplina, exigência e monitoramento e o desenvolvimento de problemas de comportamento.

Vários estudos nacionais demonstram relações importantes entre as práticas educativas utilizadas pelos pais e responsáveis e problemas de comportamento das crianças (Bolsoni-Silva \& Loureiro, 2011; Bolsoni-Silva \& Marturano, 2007; Cecconelo, De Antoni, \& Koller, 2003; Gallo \& Williams, 2010; Garcia, Brino \& Williams, 2009; Gomide, 2003; Leme, \& Bolsoni-Silva, 2010; Macarini, Martins, Minetto \& Vieira, 2010; Mondin, 2008; Padilha \& Williams, 2004; Rios \& Williams, 2010; Salvador \& Weber, 2008; Santos \& Williams, 2008; Williams, 2010). Ao analisarem as práticas educativas parentais, os trabalhos descrevem quais comportamentos os pais devem ter na sua relação com os filhos para favorecer comportamentos pró-sociais e o desenvolvimento socioemocional das crianças e diminuir o aparecimento nelas de problemas de comportamento internalizantes e/ou externalizantes. Alguns estudos, como o de Cecconelo, De Antoni e Koller (2003), destacam também indicadores nas esferas famíliar, pessoal e social (características individuais, experiência dos pais em suas famílias de origem, rede de apoio social e eventos estressantes no ciclo vital) que favorecem o engajamento dos pais em práticas educativas positivas. Não obstante, poucos estudos analisam os pensamentos dos pais a respeito de suas práticas educativas e a influência que eles podem ter na educação dos filhos.

Alguns pesquisadores consideram que os pensamentos dos pais em relação aos comportamentos de cuidar e educar crianças e ao relacionamento deles com seus filhos, descritos como cognições sociais parentais, servem para filtrar as experiências, influenciar as interpretações do comportamento, preparar o ambiente para a ação e promover mudança no comportamento (Hawk \& Holden, 2006); ou seja, as cognições dos pais acerca do comportamento de seus filhos e do relacionamento estabelecido com eles influenciam a forma como os pais e mães se sentem e se relacionam com seus filhos (Rubin \& Mills, 1992). Assim, dependendo das cognições dos pais, poderíamos ter uma probabilidade maior ou menor de eles se engajarem em comportamentos que poderiam ajudar ou prejudicar o desenvolvimento dos seus filhos, por isso este é um aspecto que é importante ser avaliado nos pais.

Mills e Rubin (1993) apresentam um modelo de processamento de informações sobre 0 comportamento parental, elaborado por Rubin, Mills e Rose-Krasnor (1989 citado em Mills \& Rubin, 1993), no qual estes apresentam os fatores que influenciam o comportamento parental: crenças parentais, fatores socioecológicos e condições pessoais e sociais. De acordo com os autores, os comportamentos dos pais resultam de um mistura dos fatores apresentados anteriormente. Assim, a crença dos pais a respeito da fase do desenvolvimento da criança, das causas do aparecimento de um determinado comportamento, da importância de certos aspectos do desenvolvimento e de como devem socializar seus filhos exercerá influência direta nas estratégias de disciplina que irão adotar na educação de seus filhos. Desse modo, as crenças parentais afetariam indiretamente 0 desenvolvimento socioemocional das crianças através do impacto nos comportamentos parentais, os quais podem ser proativos (estratégias para promover comportamentos adequados nos seus filhos) ou reativos (estratégias para eliminar problemas de comportamento de seus filhos), dependendo dos objetivos estabelecidos pelos pais. Vale destacar 
que outros fatores influenciam a relação entre comportamento parental e objetivos parentais, entre os quais se podem destacar percepções e conhecimentos sobre o desenvolvimento infantil e as características da personalidade da criança, a qualidade do relacionamento afetivo estabelecido com a criança, o bem-estar emocional dos pais e da criança e a predisposição tanto dos pais quanto da criança. Todos esses fatores também recebem influência do contexto social e emocional no qual a díade esta inserida.

Holden e Hawk (2003) desenvolveram um constructo, a metaparentagem, que busca sistematizar vários aspectos das teorias sobre a parentagem. Assim, no presente trabalho temse como objetivo descrever a metaparentagem e revisar a literatura da área.

A revisão da literatura foi realizada com as palavras-chaves metaparenting e metaparentagem, em bases de dados nacionais e internacionais (PsyclNFO, Lilacs, Sage, Medline e Google acadêmico), sendo encontrados somente trabalhos em inglês, a saber, dois artigos (Nicholson, Howard \& Borkowski, 2008; Tamm, Holden, Nakonezny, Swart e Hughes (2011), duas dissertações de mestrado (Nicholson, 2006; Vlach, 2005) e uma tese de doutorado (Hawk, 2007), os quais serão descritos a seguir. A pesquisa localizou uma dissertação de mestrado da Universidade de Hong Kong (Wong, Wai-lap, Lance, 2011), mas esta não estava disponível para download.

\section{DEFINIÇÃO DE METAPARENTAGEM}

Segundo Holden e Hawk (2003), a metaparentagem consiste em um conjunto de pensamentos de avaliação dos pais a respeito da educação dos filhos que tipicamente ocorre antes ou depois das interações entre pais e filhos. O prefixo "meta" refere-se ao conhecimento dos processos internos que resultam em uma compreensão mais abrangente da parentalidade, de tal modo que a metaparentagem consiste em pensar ou refletir a respeito das práticas parentais. Tal processo é avaliativo, no sentido de não ser automático, mas intencional, indicando alguma deliberação ou reflexão. Os pensamentos dos pais centramse na criança, em seus papéis parentais, no relacionamento pai-filho ou em considerações relacionadas, como o contexto no qual tenha ocorrido um determinado evento. Em geral, o marco da metaparentagem é que os pensamentos ocorrem fora da interação paisfilho, embora algumas cognições metaparentais possam ocorrer durante as interações. Os autores destacam que essa forma de pensamento é uma atividade mental comumente empregada pelos pais, mas por muito tempo ela foi desconsiderada pelos pesquisadores da área.

A metaparentagem ocorre de diversas maneiras. Ela pode ocorrer quando uma mãe toma uma decisão deliberativa de que seu filho já está suficientemente grande para ser desmamado, ou quando o pai tenta determinar se a agressividade do filho é um problema ou uma fase normal do desenvolvimento, ou mesmo, quando os pais param e fazem um balanço do bem-estar de seus filhos; quando debatem sobre deixar a filha adolescente ir a um baile; ou quando uma mãe tenta ajudar sua filha tímida. Todos os exemplos acima demonstram a metaparentagem em ação, ou seja, os pais estão envolvidos em uma atividade cognitiva abstrata que leva em conta considerações a respeito da criança fora da interação em progresso (Holden. \& Hawk, 2003).

\section{COMPONENTES DA METAPARENTAGEM}

Por questões heurísticas e científicas, a metaparentagem foi separada em quatro componentes, embora estes estejam intimamente relacionados e muitas vezes se misturem (Hawk \& Holden, 2006; Holden. \& Hawk, 2003), a saber: antecipação, avaliação, resolução de problemas e reflexão.

1. Antecipação são considerações intencionais dos pais sobre algo que ainda não ocorreu na fase de desenvolvimento da criança (por exemplo, proteger as tomadas da casa antes de a criança começar a engatinhar). Este componente consiste de antecipações a curto e longo prazo e pode envolver aspectos relacionados à criança, aos pais ou ao relacionamento.

2. Avaliação é a aferição dos pais em relação à criança, a si mesmo e ao contexto (por exemplo, monitorar o relacionamento da criança com os pares). É um esforço do pai para saber o que está acontecendo com os filhos e com os cuidados deles. Podem ocorrer a avaliação da criança, a autoavaliação e a avaliação do contexto. 
3. Resolução de problemas - envolve diversos aspectos dos pensamentos parentais, incluindo identificar o problema, planejar uma solução, implementar a solução e avaliar o resultado. Os pontos centrais desse domínio são: reconhecer o problema, ao invés de ignorá-lo ou evitá-lo; identificar a fonte do problema; gerar possíveis soluções; antecipar possíveis resultados associados às soluções; testar uma solução e avaliar o sucesso dessa solução.

4. Reflexão é o reasseguramento dos pais quanto ao seu próprio comportamento, ao comportamento da criança ou a interações passadas entre a criança e os pais, o que pode contribuir para avaliar fatores relacionados à educação da criança de uma maneira racional. Tal componente ajuda o indivíduo a identificar as características de como os pais foram criados, quais comportamentos desejam empregar e quais querem evitar na relação atual com os seus filhos. Podem-se também ter reflexões com um foco mais a curto-prazo, caracterizado por reflexões gerais sobre as tendências do desenvolvimento infantil ou o funcionamento da família. Embora tal componente seja relacionado à avaliação, a diferença é que ele inclui considerações a longo prazo e os seus resultados são menos diretamente relacionados a um comportamento especifico ou a um conjunto de comportamentos, visto que consiste em reflexões; mas pode também estar relacionado à antecipação, pois o comportamento reflexivo no qual os pais se engajam pode levá-los a estabelecer objetivos a longo-prazo para os seus filhos e para os seus próprios comportamentos parentais.

Holden e Hawk (2003) ressaltam que, pelo fato de a metaparentagem ser primeiro e principalmente um processo interno ou encoberto de pensamento, o constructo não pode ser observado diretamente; todavia, geralmente os pensamentos metaparentais levam a manifestações externas, como por exemplo, falar com o(a) companheiro(a), parentes ou amigos sobre preocupações ou problemas enfrentados com o(a) filho(a).

\section{FUNÇÕES DA METAPARENTAGEM}

Segundo Holden e Hawk (2003), a metaparentagem tem três funções centrais para os pais: (1) promover uma parentagem efetiva: diante dos contextos mais desafiadores, os pais que empregam resolução de problemas e buscam suporte conseguem diminuir o estresse, o que os ajuda a adaptarem-se à situação; a falta de sensibilidade aos problemas dos filhos pode reduzir a probabilidade de ações adequadas, ou seja, um pai/mãe engajado em metaparentagem consegue perceber o início de um problema, enquanto um pai/mãe que não pratica a metaparentagem pode ignorá-lo, falhar ou talvez negar sua existência; (2) promover relações sociais e suportes sociais ao levar os pais a procurarem ajuda em relação a questões cognitivas (informações adicionais, conselhos, assistência, etc.) ou afetivas (reasseguramento, conforto, encorajamento, etc.), de tal modo que pode ocorrer uma redução no estresse relacionado aos cuidados com as crianças; (3) levar a transformações parentais (muitos dos objetivos principais dos programas de educação parental são, em última análise, designados para promover a metaparentagem).

A metaparentagem tem também funções importantes para as crianças, algumas das quais seriam a de ajudar a proteger a criança de lesões, resguardando-a de situações que seriam difíceis, e a de modelar comportamentos racionais e ponderados (Holden \& Hawk, 2003). Como afirmam Hawk e Holden (2006), a partir dos processos descritos anteriormente, vários fatores relativos à educação dos filhos podem ser lidados de maneira efetiva, ou seja, é esperado que os pais que pratiquem a metaparentagem frequentemente tenham menor probabilidade de se engajar em práticas parentais mal adaptadas ou extremas, tornando esse constructo um fator importante de se avaliar quando se pensa em prevenção de maus-tratos.

\section{AVALIAÇÃO DA METAPARENTAGEM}

Para avaliar a metaparentagem, Holden e Hawk (2006) elaboraram um questionário, o Metaparenting Questionnaire (MPQ) ${ }^{3}$, composto por 50 questões relativas aos quatro componentes do constructo (avaliação, antecipação, resolução de problemas e reflexão). Em uma investigação inicial do constructo Hawk e Holden (2006) aplicaram o Metaparenting

3 Questionario de Metaparentagem, instrumento em processo de validação transcultural por Manfrin, Williams e D’Affonseca (2011), não publicado. 
Questionnaire (MPQ) em uma amostra de 116 mães de crianças com idades variando entre quatro e seis anos. Os resultados obtidos demonstraram que as mães relataram se engajar frequentemente em metaparentagem e que as medidas obtidas eram fidedignas. Entre os fatores que prediziam a metaparentagem estavam o número de filhos, sua idade e seu gênero; ou seja, mães que tinham menos filhos relataram mais resolução de problemas, mães de meninos relataram mais antecipação e mães de crianças menores obtiveram maiores escores totais de metaparentagem. Além disso, os dados demonstraram que mães expostas a maiores níveis de estresse tinham maiores escores de reflexão.

\section{ESTUDOS SOBRE METAPARENTAGEM}

Vlach (2005) buscou comparar a influência do gênero, do tipo de trabalho exercido pelos pais e da experiência de parentagem com a metaparentagem em pais de crianças e lactentes. Sessenta pais responderam, eletrônica ou pessoalmente, um questionário no qual eles deveriam relatar com que frequência (variando de às vezes a geralmente) eles se engajavam nos quatro domínios de metaparentagem (antecipação, avaliação, reflexão e resolução de problemas). Os resultados obtidos indicaram que o gênero e o tipo de trabalho exercido pelos participantes não influenciaram significativamente os escores de metaparentagem. De forma geral, os pais disseram ter um alto grau de satisfação e de competência quando se engajavam em altos níveis de metaparentagem.

$\mathrm{Na}$ dissertação de mestrado de Nicholson (2006) a autora avaliou antecedentes e consequências da metaparentagem em uma amostra de 66 pais que participavam de um projeto longitudinal (Notre Dame Fathers' Project) do Centro de Crianças e Família da Universidade de Notre Dame, em Indiana, EUA, no qual um grupo diverso de pais foi acompanhado dos seis meses aos dois anos de idade de seus filhos. O tipo de moradia das crianças e os modelos parentais positivos foram relacionados aos modelos de processamento interno de parentagem dos pais conforme mensurados pela metaparentagem. Os resultados obtidos demonstraram que altos níveis de metaparentagem estavam correlacionados com a parentagem autoritativa $^{4}$, maior conhecimento sobre desenvolvimento infantil, menos estresse e menor potencial abusivo, quando foram controlados a idade e o nível de inteligência dos pais. De acordo com a pesquisadora, os resultados sugerem que as experiências passadas influenciam 0 modelo de processamento interno dos pais para parentagem e, consequentemente, a habilidade de realizar a metaparentagem. Além disso, a metaparengem influencia a efetividade parental.

Nicholson, Howard e Borkowski (2008) avaliaram os antecedentes e as correlações da metaparentagem em cinco subescalas (responder, prevenir, monitorar, instruir e modelar) com 74 pais. A maioria dos participantes $(n=45)$ foi recrutada de um estudo longitudinal de nivel nacional (Parenting for the First Time), o qual avaliava negligência em díades mãe/criança. Essas mães foram recrutadas em hospitais, clínicas de saúde, agências de serviço social e programas para mães em idade escolar. A amostra de mães incluía adolescentes (58\%), adultas com pouca escolaridade (consideradas pelos autores como aquelas que tinham menos de dois anos de universidade; 25\%), e mães com alta escolaridade (curso superior e pós-graduação, $17 \%$ ). As mães que participaram do projeto deveriam indicar o nome do pai biológico ou social do bebê, os quais foram recrutados para participar do Notre DameFathers' Project. Para aumentar o tamanho da amostra, os pais também foram recrutados em igrejas e centros esportivos da comunidade $(n=10)$, assim como de outros estudos sobre parentagem desenvolvidos pelo Notre Dame Center for

\footnotetext{
Pais democráticos respeitam a individualidade, mas também enfatizam valores sociais. Eles têm confiança em sua capacidade de orientar as crianças, mas também respeitam as decisões, os interesses, as opiniões e a personalidades delas. São afetuosos e acolhedores, mas também exigem bom comportamento, são firmes na manutenção de padrões e estão dispostos a impor castigos limitados e criteriosos quando necessário, no contexto de um relacionamento afetuoso e positivo. Explicam a base de suas opiniões e estimulam a troca de ideias. Seus filhos sentem-se seguros, tanto por saberem que são amados quanto por saberem o que se espera deles. Tendem a ser mais independentes, autocontrolados, seguros, exploradores e satisfeitos (Papalia, Olds \& Feldman, 2009)
} 
Children and Families ( $\mathrm{n}=19$ ), os quais avaliavam mães tanto de alto quanto de baixo risco. Dos 127 pais triados inicialmente, 101 foram contactados e 87 aceitaram participar da pesquisa. Estes últimos tinham um nível de escolaridade um pouco maior, maior probabilidade de estarem casados com a mãe da criança e maiores níveis de envolvimento com os filhos, quando comparados com os pais que se recusaram a participar da pesquisa. Os pais foram entrevistados quando seus filhos tinham seis, doze, dezoito e vinte e quatro meses no Notre Dame Fathers' Project, e no estudo analisado foram utilizadas as informações obtidas na coleta realizada com seis, doze e dezoito meses. Os pais responderam a uma série de instrumentos: aos seis meses de idade do filho foram avaliadas as características demográficas, comportamentos de apego e a história de vida parental. Aos doze e dezoito meses os pais responderam a uma entrevista de metaparentagem elaborada pelos autores (RPM3 - 19 questões semiestruturadas para avaliar os seguintes aspectos: responder, prevenir, monitorar, acompanhar e modelar), e medidas de conhecimento parental, estilo parental, estresse parental e potencial de abuso infantil. Um modelo de equação estrutural revelou que os relatos de papel parental positivo dos pais e inteligência foram relacionados a modelos de atuação de parentagem medidos nos cinco componentes da metaparentagem avaliados. Pais com modelos positivos, que residiam com seus filhos e tinham altos níveis de metaparentagem, tinham características demográficas associadas com melhores índices de parentagem e desenvolvimento da criança. Além disso, os autores encontraram que níveis maiores de metaparentagem estavam associados à parentagem autoritativa e a menor potencial de abuso.

Em sua tese de doutorado, Hawk (2007) buscou compreender a relação entre diferentes constructos de cognições sociais para investigar por que alguns pais são cuidadores mais sensíveis e efetivos do que outros. Um dos objetivos foi examinar a relação entre cinco variáveis sociocogntivas: conceito de desenvolvimento, insight, apego seguro, metaparentagem e Mindfulness. $O$ segundo objetivo foi examinar as associações entre os constructos de cognição social, parentagem efetiva medida pela parentagem autoritativa $e$ um constructo maior de cuidados infantis efetivos (AWARE parenting ${ }^{5}$ ), e três avaliações centradas na criança (relações calorosas entre pais e filhos, história de agressões e ferimentos). Participaram da pesquisa cento e duas mães, a maioria com formação superior. As participantes responderam onze medidas de autorrelato sobre sua parentagem com foco na criança. Setenta e quatro amigos ou membros da família participaram como informantes secundários e forneceram dados sobre a metaparentagem das mães e o programa de parentagem consciente (AWARE parenting). Os dados obtidos demonstram que muitos dos constructos individuais estavam associados entre si. $O$ constructo da metaparentagem, quando extraído do fator de análise, foi relacionado a um número de medidas de parentagem e de medidas relacionadas à criança maior do que qualquer outro constructo utilizado na pesquisa. Além disso, todos os três fatores de cognição social obtiveram correlações significativas com as duas medidas de parentagem; ou seja, aqueles pais que tiveram escores maiores em ideias sobre os cuidados com as crianças, interação receptiva e metaparentagem, também relataram mais comportamentos democráticos e parentagem consciente (AWARE parenting).

Em relação às variáveis das crianças, a metaparentagem esteve relacionada a comportamentos agressivos e de proximidade das crianças, e a interação receptiva também foi um preditor dos escores de agressão das crianças. Mães que tiveram a maior pontuação em interação receptiva relataram menores escores de agressão das crianças. As lesões na criança não foram relacionadas a nenhum dos fatores analisados. Os dados dos informantes secundários foram relacionados

5 Parentagem consciente (Aware Parenting) é uma filosofia de cuidados da criança baseada no trabalho da Dr. Aletha Solter a qual utiliza alguns fatores do estilo democrático de Baurind com disciplina não punitiva e receptividade emocional (Bronstein et al., 1996). O programa visa trabalhar com os pais os seguintes componentes: suporte (elogiar, concordar, encorajar e demonstrar afeição), atenção (ouvir, favorecer diálogo e individualidade), responsividade (reconhecer as necessidades que a criança expressa, responder a essas necessidades quando possível e ver a perspectiva da criança). Além disso, o programa sugere orientação (fornecer informação e diretrizes apropriadas sobre valores e comportamentos culturais) e promover receptividade as emoções (permitir que a criança expresse abertamente suas emoções). 
significativamente à parentagem consciente (AWARE parenting), mas não com a metaparentagem (Hawk, 2007).

Tamm, Holden, Nakonezny, Swart e Hughes (2011) investigaram a metaparentagem em pais de crianças com sintomas de TDAH, incluindo preditores, correlatos e resultados de intervenções. Participaram da pesquisa 68 pais (63 mães e cinco pais) de crianças ( $M=5.18$ anos \pm 1.33 ; $69 \%$ meninos) com sintomas significativos de TDAH (i.e., mais de seis sintomas de desatenção ou hiperatividade/impulsividade ocorrendo em dois ou mais ambientes, mesmo sem diagnóstico médico) que estavam inscritos em um programa de treinamento comportamental conduzido como parte de um projeto de pesquisa da University of Texas Southwestern Medical Center. Os participantes foram recrutados por meio de encaminhamentos e anúncios e por correspondência. Os pais eram predominantemente brancos, com média de idade de $37.82 \pm 5.78$ anos, e a maioria tinha cursado mais do que o Ensino Médio. Apos uma triagem inicial, os pais participaram de um programa de treinamento parental intitulado Programa Educacional Parental Comunitário (Community Parent Education Program -COPE) (Cunningham et al., 1998), o qual ocorria semanalmente ( $n=10$ semanas), com duas horas de duração em cada encontro. Os tópicos abordados no treinamento incluíam estratégias de parentagem (prestar atenção em comportamentos positivos, ignorar os negativos, implementar sistema de recompensa e aprender a lidar com comportamento desafiador). $\mathrm{Na}$ primeira e na ultima das sessões do treinamento os pais preencheram instrumentos que avaliavam a metaparentagem, 0 estresse parental, práticas parentais e sintomas de TDAH dos filhos. Analisou-se a relação entre a metaparentagem e preditores de base, bem como se a metaparentagem previa: (1) comportamentos dos pais no início do estudo, (2) atritos e (3) 0 estresse dos pais e 0 comportamento dos pais com os filhos. Os resultados obtidos demonstraram que mães com maior escolaridade, com menos pessoas vivendo em casa e maiores níveis de estresse parental relataram mais metaparentagem. Pais com escores menores em resolução de problemas e avaliação relataram mais parentagem inconsistente. Os pais que apresentaram maiores escores em resolução de problemas tinham uma probabilidade maior de abandonar o treinamento. Escores mais altos em resolução de problemas e reflexão na linha de base foram associados a maior estresse parental. Maiores índices de reflexão na linha de base previram como resultado sintomas de hiperatividade e impulsividade nas crianças. Segundo os autores, os resultados encontrados indicam que a metaparentagem esta associada tanto com os comportamentos parentais quanto com a tomada de decisão em completar ou não o treinamento de pais. Além disso, a metaparentagem aparenta ser um constructo complexo, finamente matizado, com associações positivas e negativas aos relatos de práticas parentais e estresse.

No Brasil, D'Affonseca, Lopes \& Williams (2010) realizaram uma pesquisa preliminar, ainda não publicada, a qual contou com a participação de catorze mulheres (26 a 53 anos, $M=36,6 ; d p=7,6)$ com pelo menos um filho de três a doze anos $(M=7,7, d p=2,8)$, a maioria com Ensino Médio completo, sendo que $50 \%$ das participantes tinham renda familiar superior a cinco salários mínimos (SM), 35,7\% tinham renda mensal de dois a três SMs e $14,5 \%$ de quatro a cinco SMs. Todas as mães responderam à versão traduzida e adaptada do Questionário de Metaparentagem (D'Affonseca, 2010), composto por vinte e quatro questões relativas aos quatro componentes do constructo: antecipação (cinco itens), avaliação (seis itens), reflexão (seis itens) e resolução de problemas (sete itens). Os dados obtidos demonstraram que todas as mães praticavam a metaparentagem e que, em média, elas praticavam mais a resolução de problemas $(M=39,5, d p=23,8)$, vindo a seguir, por ordem decrescente, a avaliação $(M=34,1, d p=19)$, a reflexão $(M=31,9, \quad d p=22,5)$ e a antecipação $(M=25,1, d p=14,2)$. Tais dados se assemelham aos obtidos por pesquisas realizadas em outros países e parecem indicar que as mães passam maior parte do tempo procurando soluções para um problema que esteja ocorrendo com o filho (resolução de problema) do que buscando ter comportamentos que evitem o aparecimento desses problemas (reflexão, antecipação); ou seja, o foco dos pensamentos parentais tende a se centrar no problema atual e menos na prevenção de problemas futuros.

Para finalizar, embora ainda existam poucos estudos na literatura internacional e nacional a respeito da metaparentagem, as pesquisas apresentadas acima sugerem que a 
metaparentagem pode ser um constructo útil e importante de ser avaliado quando se trabalha com famílias, mais especificamente com 0 relacionamento de mães/pais com seus filhos, visto que esse relacionamento está correlacionado com a parentagem autoritativa, a competência parental e menor potencial de abuso. Por ser uma área de estudos recentes, a maioria dos achados referemse à população norte-americana, branca e com algum grau de escolaridade (em todas as pesquisas os participantes tinham cursado alguns anos do ensino regular). Haveria diferenças na metaparentagem de pais de diferentes culturas? Haveria diferenças significativas entre a metaparentagem em pais/mães com diferentes graus de escolaridade e níveis socioeconômicos? Novos estudos na área, com amostras maiores de diferentes culturas, níveis de escolaridade, configuração familiar e níveis socioeconômicos contribuiriam para aprofundar a compreensão do fenômeno e poderiam ser um recurso útil para a área de intervenção junto a famílias e prevenção de problemas de comportamento infantil.

Além disso, pesquisas futuras, com amostras maiores de participantes, poderão investigar se a influência de algumas variáveis presentes na educação dos filhos (problemas de disciplina, características da criança) ou variáveis da mãe (problemas no relacionamento conjugal, problemas de saúde mental, estresse parental) influenciam a metaparentagem.

\section{REFERÊNCIAS}

Bolsoni-Silva, A. T. \& Loureiro, S.R. (2011). Práticas educativas parentais e repértório comportamental infantil: comparando crianças diferenciadas pelo comportamento. Paidéia, 21 (48), 61-71.

Bolsoni-Silva, A. T., \& Marturano, E. M. (2007). A qualidade da interação positiva e da consistência parental na sua relação com problemas de comportamento de préescolares. Interamerican Journal of Psychology, 41, 349358.

Cecconelo, A.M.; De Antoni, C. \& Koller, S.H. (2003). Práticas educativas, estilos parentais e abuso físico no contexto famíliar. Psicologia em estudo, Maringá, 8, 4554.

D'Affonseca, S.M.; Lopes, N.R.L. \& Williams, L.C.A. (2010). Avaliando a metaparentagem de mães brasileiras: dados preliminares (Resumo). Em: Sociedade Brasileira de Psicologia (org.) Resumos de comunicação científica. XL Reunião Anual da Sociedade Brasileira de Psicologia, 20 a 23 de outubro, Curitiba-PR.

Gallo, A.E. \& Williams, L.C.A. (2010). Ensino de habilidades parentais a mães de adolescentes em conflito com a lei. Em L.C.A. Williams, J.M.D. Maia \& K.S.A. Rios. (Orgs.)
Aspectos psicológicos da violência: Pesquisa e intervenção cognitivo-comportamental. (pp. 407-427). Santo André: ESETec.

Garcia, S.C., Brino, R. \& Williams, L.C.A. (2009). Risco e resiliência em escolares: Um estudo comparativo com múltiplos instrumentos. Psicologia da Educação, 28, 2349.

Gomide, P.I.C. (2003). Estilos Parentais e comportamento anti-social. In A. Del Prette \& Z. Del Prette (Orgs.). Habilidades sociais, desenvolvimento e aprendizagem: questões conceituais, avaliação e intervenção (pp.2160). Campinas: Alínea.

Guralnick, M. J. (1998). Effectiveness of early intervention for vulnerable children: A developmental perspective. American Journal of Mental Retardation, 102 (4), 319345.

Hawk, C. K. (2007). The social-cognitive underpinnings of effective caregiving. Tese de doutorado, Programa de Pós Graduação em Filosofia, Universidade do Texas, Austin. Disponível em http://hdl.handle.net/2152/13245.

Hawk, C.K. \& Holden, G.W. (2006). Meta-parenting: an initial investigation into a new parental social cognition construct. Parenting: Science and Practice,6, (4), 321-34.

Holden, G. W., \& Hawk, C. (2003). Meta-parenting in the journey of child rearing: A mechanism for change. Em: L. Kuczynski (Ed.), Handbook of dynamics in parent-child relations. Thousand Oaks, CA: Sage.

Leme, V. B. R. \& Bolsoni-Silva, A. T. (2010). Habilidades Sociais Educativas Parentais e comportamentos de préescolares. Estudos de Psicologia, 15 (2),161-173.

Macarini, S. M.; Martins, G. D. F.; Minetto, M. F. J. \& Vieira, M. L. (2010). Práticas parentais: uma revisão da literatura brasileira. Arquivos Brasileiros de Psicologia, 62 (1), pp. 119-134.

Mills, R.S.L., \& Rubin, K.H. (1993). Parental beliefs about children's social development. Em: S. Duck (Ed.), Understanding Relationship Processes, Vol. 2, Learning about relationships (pp. 98-117). Newbury Park, CA: SagePublications.

Mondin, E.M.C. (2008). Práticas educativas parentais e seus efeitos na criação dos filhos. Psicologia Argumento , 26, p. 233-244

Nicholson, J. Howard, K. S.; Borkowski, J. G. (2008). Mental models for parenting: correlates of metaparenting among fathers of young children. Fathering, 6(1), 39-61.

Nicholson, J.S. (2006). Precursors to Paternal Identity: Antecedents and Consequences of Metaparenting. Dissertação de mestrado não publicada, Programa de Pós-Graduação em Psicologia, Universidade de Notre Dame, Indiana, EUA.

Padilha, M. G. S. \& Williams, L.C.A. (2004). Considerações sobre estilos parentais de pais que cometem abuso físico, psicológico ou sexual contra seus filhos. Em M.Z.S. Brandão, F.C.S. Conte, F.S. Brandão, Y.K. Ingberman, V.L. M. Silva \& S.M. Oliani. Sobre comportamento e cognição: Estendendo a psicologia comportamental e cognitiva aos contextos da saúde, das organizações, das relações pais e filhos e das escolas, v. 14, (pp.286-291). Santo André: ESETec. 
Papalia, D.E; Olds, S.W; Feldman, R. D. (2009) Desenvolvimento humano. Tradução: José Carlos Barbosa, Carla Versace e Mauro Silva. 10. ed. São Paulo: McGraw - Hill.

Rios, K.S.A. \& Williams, L.C.A. (2010). Um programa de intervenção sistemática com famílias de baixa renda para prevenção de comportamentos-problemas em crianças. Em L.C.A. Williams, J.M.D. Maia \& K.S.A. Rios. (Orgs.) Aspectos psicológicos da violência: Pesquisa e intervenção cognitivo-comportamental. (p.p. 381-406). Santo André: ESETec.

Rubin, K.H. \& Mills, R.S.L. (1992). Parents' ideas about the development of aggression and withdrawal. Em: I. Sigel, J. Goodnow, \& A. McGillicuddy-deLisi (Eds.), Parental Belief Systems (pp. 41-68). Hillsdale, NJ: Erlbaum.

Salvador, A.P.V. \& Weber, L.N.D. (2008). A relação entre práticas educativas e estilos parentais como 0 desenvolvimento de crianças e adolescentes. Em: Weber, L.N.D. (org.) Família e desenvolvimento: visões interdisciplinares. Curitiba: Juruá.

Santos, G.E. \& Williams, L.C.A. (2008). Prevenção terciária de problemas de comportamento infantil: Intervenção com pais que maltratam.Em E.G. Mendes, M.A. Almeida, \& M.C. P. I. Hayashi (Orgs.). Temas em Educação Especial: Conhecimentos para fundamentar a prática.(pp.213-226).Araraquara: Junqueira\& Marin Editores/CAPES PROESP.

Tamm, L.; Holden, G.W.; Nakonezny, P.A.; Swart, S. \& Hughes, C.W. (2011) . Metaparenting: associations with parenting stress, child-rearing practices, and retention in parents of children at risk for ADHD. $A D H D$ AttentionDeficitandHyperactivityDisorders, 4(1), 1-10.

Tucunduva, C. \& Weber, L.N.D. (2008) Práticas educativas parentais: fatores de risco e de proteção ao desenvolvimento dos filhos. Em: Weber, L.N.D. (org.) Família e desenvolvimento: visões interdisciplinares. Curitiba: Juruá.
Vlach, J. L. (2005) Meta-Parenting in Parents of Infants and Toddlers. Dissertação de mestrado não publicada. Toulouse School of Graduate Studies, Universidade Norte do Texas, Denton, Texas, EUA.

Weber, L. N. D.; Prado, P. M.; Viezzer, A. P. \& Brandenburg, O. J. (2004). Identificação de estilos parentais: o ponto de vista dos pais e dos filhos. Psicologia: Reflexão e Crítica, 17(3), 323-331.

Weber, L.N. D.; Viezzer, A.P.; Brandenburg, O. J. (2004) O uso de palmadas e surras como prática educativa. Estudos de Psicologia, 9(2), 227-237.

Williams, L.C.A. (2010). A família como um agente transformador da violência: empregando práticas positivas. Em: L.C.A. Williams, R.C. Padovani, Araújo, E.A.C., A.C. Stelko-Pereira, G.R. Ormeño \& E. Eisestein. (Orgs.). Fortalecendo a rede de proteção da criança e do adolescente. (pp. 55-57).São Carlos: Pedro e João Editores.

Wong, Wai-lap, Lance, (2011). How do parents think about and evaluate childrearing issues? : exploring patterns of meta-parenting and their characteristics. Dissertação de Mestrado não publicada, Programa de Pós-Graduação em Filosofia, Universidade de Hong Kong (Pokfulam Road, Hong Kong).

Endereço para correspondência: Sabrina Mazo D Affonseca. Rua Joaquim Augusto Ribeiro de Souza, 889, CEP: 13563330, São Carlos-SP, Brasil. E-mail: samazo@hotmail.com. 